\title{
Matrix metalloproteases as maestros for the dual role of LPS- and IL-10-stimulated macrophages in cancer cell behaviour
}

Ana P. Cardoso ${ }^{1,2}$, Marta L. Pinto ${ }^{1,3}$, Ana T. Pinto ${ }^{1,2}$, Marta T. Pinto ${ }^{4}$, Cátia Monteiro ${ }^{1}$, Marta I. Oliveira1, Susana G. Santos ${ }^{1,2}$, João B. Relvas ${ }^{5}$, Raquel Seruca ${ }^{4,6}$, Alberto Mantovani ${ }^{7,8}$, Marc Mareel ${ }^{9}$, Mário A. Barbosa ${ }^{1,3}$ and Maria J. Oliveira ${ }^{1,6^{*}}$

\begin{abstract}
Background: The interactions established between macrophages and cancer cells are largely dependent on instructions from the tumour microenvironment. Macrophages may differentiate into populations with distinct inflammatory profiles, but knowledge on their role on cancer cell activities is still very scarce. In this work, we investigated the influence of pro-inflammatory (LPS-stimulated) and anti-inflammatory (IL-10-stimulated) macrophages on gastric and colorectal cancer cell invasion, motility/migration, angiogenesis and proteolysis, and the associated molecular mechanisms.

Methods: Following exposure of gastric and colon cancer cell lines to LPS- and IL-10-stimulated human macrophages, either by indirect contact or conditioned media, we analyzed the effect of the different macrophage populations on cancer cell invasion, migration, motility and phosphorylation status of EGFR and several interacting partners. Cancer-cell induced angiogenesis upon the influence of conditioned media from both macrophage populations was assessed using the chick embryo chorioallantoic membrane assay. MMP activities were evaluated by gelatin zymograhy.

Results: Our results show that IL-10-stimulated macrophages are more efficient in promoting in vitro cancer cell invasion and migration. In addition, soluble factors produced by these macrophages enhanced in vivo cancer cell-induced angiogenesis, as opposed to their LPS-stimulated counterparts. We further demonstrate that differences in the ability of these macrophage populations to stimulate invasion or angiogenesis cannot be explained by the EGFR-mediated signalling, since both LPS- and IL-10-stimulated macrophages similarly induce the phosphorylation of cancer cell EGFR, c-Src, Akt, ERK1/2, and p38. Interestingly, both populations exert distinct proteolytic activities, being the IL-10-stimulated macrophages the most efficient in inducing matrix metalloprotease (MMP)-2 and MMP-9 activities. Using a broad-spectrum MMP inhibitor, we demonstrated that proteolysis was essential for macrophage-mediated cancer cell invasion and angiogenesis.
\end{abstract}

Conclusions: We propose that IL-10- and LPS-stimulated macrophages distinctly modulate gastric and colorectal cancer cell behaviour, as result of distinct proteolytic profiles that impact cell invasion and angiogenesis.

Keywords: Tumour microenvironment, M1 and M2-like macrophages, Invasion, Angiogenesis, Gastrointestinal cancer, MMPs

\footnotetext{
* Correspondence: mariajo@ineb.up.pt

'i3S-Instituto de Investigação e Inovação em Saúde/INEB-Institute of

Biomedical Engineering, University of Porto, Porto, Portugal

${ }^{6}$ Department of Pathology and Oncology, Faculty of Medicine, University of

Porto, Porto, Portugal

Full list of author information is available at the end of the article
} 


\section{Background}

Solid tumours are complex entities with several cellular constituents other than malignant cells. Macrophages constitute a major component of the immune infiltrate in these tumours and are known to interact with cancer cells and to play a crucial role in distinct steps of cancer progression, such as survival, immune evasion, migration, invasion and metastasis [1-4]. The presence of macrophages is usually an indicator of poor prognosis in many types of malignancies $[2,5]$. In colorectal cancer, tumour-associated macrophages (TAMs) have been frequently correlated with better prognosis $[5,6]$, although some studies refer that disease outcome may vary according to macrophage molecular profile and localization within the tumour [6-8]. In gastric cancer, few reports point TAMs as positive predictors of patient survival $[9,10]$, while most studies associate high macrophage densities with tumour promotion and worse overall survival $[5,11,12]$. Previous studies revealed that macrophages stimulate breast cancer cell migration and invasion through a paracrine loop involving colonystimulating factor-1 (CSF-1), produced by cancer cells, and EGF produced by macrophages $[1,13]$. Our own work recently reinforced these studies, describing that gastric and colorectal cancer cell motility, proteolysis and invasion are stimulated by macrophages and that epidermal growth factor (EGF) is a key molecule in this crosstalk [14]. However, nothing was described about the putative impact of different macrophage subpopulations in gastric and colorectal cancer cell properties and signalling.

Macrophages are highly plastic and very versatile in response to microenvironment stimuli, including cues released by neoplastic cells [15-17]. Despite their intermediate activation state, macrophages are generally classified into two main functional phenotypes, reflecting the Th1/Th2 response of CD4 T helper cells [18-20]. M1 macrophages are induced by interferon-gamma (IFN- $\gamma$ ), microbial products, such as lipopolysaccharides (LPS) and cytokines, like tumour necrosis factor-alpha $(T N F-\alpha)[21]$. They are generally characterized by inflammatory, microbicidal and tumoricidal activities, high antigen presenting capacity, high secretion of IL-12, IL23 , IL-6, nitric oxide (NO) and reactive oxygen intermediates (ROI) and low IL-10 production [22]. On the other hand, M2 and M2-like macrophages polarize in response to IL-4 and IL-13, IL-10 or glucocorticoid hormones and are generally described to present low IL-12 and IL-6 and high IL-10 expression, as well as an increased ability to scavenge, repair and remodel tissue, promoting angiogenesis and tumour progression [22, 23]. Although most current studies suggest TAMs as being a skewed M2-like macrophage population, engaging in cancer promoting activities, their phenotype can vary according to their distribution within the tumour $[19,24-$ 26]. Therefore, clarifying the role of distinct macrophage subsets in cancer and unravelling the concomitant molecular mechanisms will contribute to the identification of novel therapeutic targets and biomarkers useful for patient stratification.

In the present work, we studied LPS- and IL-10stimulated macrophages modulation of gastric and colorectal cancer cell-related activities, such as invasion, proteolysis, motility, migration and angiogenesis and determined the associated molecular mechanisms. Overall, our results demonstrate that distinct proteolytic activities of these macrophage populations differently modulate the behaviour of gastric and colorectal cancer cells, providing new insights for the development of new and more efficient anti-tumour therapies.

\section{Methods}

\section{Cell culture and reagents}

AGS (CRL-1739) and RKO (CRL-2577) cells, derived respectively from a human diffuse gastric and colon carcinoma, were purchased from the American Type Culture Collection (ATCC, Manassas, VA, USA) in 2012. Cell lines were tested and authenticated by autosomal STR DNA profiling, in which a DNA sample was analysed with POWERPLEX 16 HS kit (Promega, Madison, WI, USA). The cell lines were last tested and authenticated on May $20^{\text {th }} 2014$, by a laboratory accredited by the College of American Pathologists and with a Quality Management System certified in accordance with NP EN ISO 9001:2008 (IPATIMUP Diagnostics, Porto, Portugal). Cells were cultured at $37{ }^{\circ} \mathrm{C}$ and $5 \% \mathrm{CO}_{2}$ humidified-atmosphere in RPMI1640 medium (Invitrogen, Merelbeke, Belgium), supplemented with $10 \%$ fetal bovine serum (FBS) (Lonza, Basel, Switzerland), $100 \mathrm{U} / \mathrm{ml}$ penicillin and $100 \mu \mathrm{g} / \mathrm{ml}$ streptomycin (Invitrogen).

\section{Human monocyte isolation and macrophage differentiation}

Human monocytes were isolated from healthy blood donors as previously described [14]. For monocyte to macrophage differentiation, $10^{6}$ monocytes $/ \mathrm{ml} / 3,8 \mathrm{~cm}^{2}$ were then cultured for 10 days in RPMI1640 medium, supplemented with $10 \% \mathrm{FBS}$ and $100 \mathrm{U} / \mathrm{ml}$ penicillin and $100 \mu \mathrm{g} / \mathrm{ml}$ streptomycin, in absence of M-CSF or other exogenous factors. LPS- and IL-10-stimulated macrophages were obtained by adding $10 \mathrm{ng} / \mathrm{ml} \mathrm{LPS}$ (Sigma-Aldrich) or IL-10 (ImmunoTools, Friesoythe, Germany), respectively, for additional $72 \mathrm{~h}$. Unstimulated (naïve) macrophages were maintained with renewed medium and used as control. All experimental protocols were conducted following the approval and recommendations of the Ethics Comittee for Health from Centro Hospitalar S. João (Porto - References 259/11 and 260/11). 


\section{Flow cytometry}

For cell surface receptor expression analysis, unstimulated, LPS - and IL-10-stimulated macrophages were harvested, by incubation with PBS-5 mM EDTA, for $30 \mathrm{~min}$ at $37{ }^{\circ} \mathrm{C}$. Macrophages were then resuspended in FACS buffer (PBS, $2 \%$ FBS, $0.01 \%$ sodium azide), and stained with antihuman CD14-FITC, HLA-DR-PE (ImmunoTools) and CD163-PE (R\&D Systems, Minneapolis, MN, USA), for 30 min at $4{ }^{\circ} \mathrm{C}$ in the dark. Isotype-matched antibodies were used as negative controls, to define background staining. Cells were acquired on a FACSCalibur ${ }^{\mathrm{TM}}$ Flow Cytometer (BD Biosciences), using Cell Quest Software (collecting 10 000 cells). Analysis was performed with FlowJo software. Percentage of positive cells was calculated by subtracting the respective isotype control. Experiments were performed with cells from at least five different donors.

\section{Enzyme-linked immunosorbent assay (ELISA)}

TNF- $\alpha$, IL- 6 and IL-10 cytokines, present in conditioned media (CM) from unstimulated, LPS- and IL-10stimulated macrophages, were quantified by ELISA according to manufacturer's instructions (BioLegend, San Diego, CA, USA) [14].

\section{Invasion assays}

Invasion assays were performed as previously [14], using BD BioCoat ${ }^{\mathrm{TM}}$ Matrigel $^{\mathrm{TM}}$ Invasion Chambers (BD Biosciences, Madrid, Spain) and AGS or RKO cells in the upper compartment, and LPS- (LPSmac) or IL-10-stimulated macrophages (IL-10mac) in the lower compartment. To discard any influence of soluble factors released along macrophage differentiation, media was renewed before invasion assays. The broad MMP inhibitor Galardin (Calbiochem, Nottingham, UK) was used at a final concentration of $10 \mu \mathrm{M}$. The invasive ratio was calculated as the ratio between the number of invasive cells in the test condition and the number of invasive cells in the control condition.

\section{Conditioned media preparation}

At the end of Matrigel $^{\mathrm{TM}}$ invasion assays, CM of cancer cells (CMMat(AGS)), LPS- (CMMat(LPSmac)) or IL-10stimulated (CMMat(IL-10mac)) macrophages or cancer cells cultured in the presence of LPS- (CMMat(AGS + LPSmac)) or IL-10-stimulated (CMMat(AGS + IL-10mac)) macrophages were collected. The influence of soluble factors produced by LPS- (CM(LPSmac)) and IL-10stimulated (CM(IL-10mac)) macrophages, in the absence of ECM components (without Matrigel ${ }^{\mathrm{TM}}$ ) were also prepared.

\section{Immunocytochemistry}

To evaluate macrophage morphology and cytoskeleton organization, $19 \times 10^{4}$ monocytes $/ \mathrm{cm}^{2}$ were seeded on glass coverslips upon isolation, and left for 10 days in culture. Treatments with LPS and IL-10 were performed as described above. To investigate the effect of distinct macrophage populations on cancer cell motility and EGFR phosphorylation, $2.7 \times 10^{4}$ AGS cells $/ \mathrm{cm}^{2}$, seeded on glass coverslips and maintained at $37{ }^{\circ} \mathrm{C}, 5 \% \mathrm{CO}_{2}$, were treated or not with CM from LPS- (CM(LPSmac)) or IL-10-stimulated macrophages (CM(IL-10mac)) for 1 or $6 \mathrm{~h}$. In parallel, RPMI media (RPMI) was used as control. Cells were immunostained for phosphoEGFR (Tyr1086), $\alpha$-tubulin and F-actin and analysed as previously described [14].

\section{Calculation of macrophage aspect ratio}

Macrophage aspect ratio was quantified using ImageJ software on images of actin/tubulin unstimulated, LPSor IL-10-stimulated macrophages. Aspect ratio was calculated as the quotient between the length of each cell major and minor axes, as previously described [27]. At least 100 cells per donor/per condition were scored, and at least three independent experiments were analysed with cells from three different donors.

\section{Quantification of motility-associated structures}

Filopodia, lamellipodia and stress fibers were quantified using ImageJ software on images of actin/tubulin regarding AGS cells treated for $6 \mathrm{~h}$ with RPMI or CM from LPS- or IL-10-stimulated macrophages. The percentage of cells with these structures was calculated considering the total cell number. At least 100 cells per donor/per condition were scored, and at least three independent experiments were analysed with cells from three different donors

\section{Timelapse microscopy}

To determine the effect of LPS- and IL-10-stimulated macrophages on cancer cell migration, $5 \times 10^{4}$ AGS cells $/ \mathrm{cm}^{2}$ were seeded. Immediately before each experiment, cells were treated with CM from LPS- (CM(LPSmac)) or IL-10stimulated (CM(IL-10mac)) macrophages (1/3 total volume) or equivalent RPMI medium (RPMI), as control. Cell trajectories followed for $13 \mathrm{~h}$ were quantified as previously described [14].

\section{Gelatin zymography}

MMP activity of LPS- and IL-10-stimulated macrophages, and of co-cultures of AGS cells with both macrophage populations was investigated by analysing CM from invasion assays through gelatin zymography, as previously described $[14,28]$.

\section{Angiogenesis assay}

Fertilized chicken (Gallus gallus) eggs obtained from commercial sources (Pintobar, Braga, Portugal) were incubated at $38{ }^{\circ} \mathrm{C}$. At day 3 of incubation, a window was opened in the shell, and 2-2.5 $\mathrm{ml}$ albumen was removed. The window was sealed with adhesive tape, and the egg re- 
incubated. At day 10 of incubation, a $3 \mathrm{~mm}$ silicon ring was placed on the growing chorioallantoic membrane (CAM), under sterile conditions. Then, $1 \times 10^{6}$ AGS cells in RPMI medium and $1 \times 10^{6}$ AGS cells in CM from LPS-treated (CM(LPSmac)) or IL-10-stimulated macrophages (CM(IL$10 \mathrm{mac}))$, with or without Galardin $(30 \mu \mathrm{M})$, were incubated within two separate rings. The window was resealed, and $72 \mathrm{~h}$ after inoculation, rings were removed, and the CAM was excised and photographed ex ovo under a stereoscope (Olympus; SZX16 coupled DP71 camera). The number of new vessels $(<20 \mu \mathrm{m}$ diameter $)$ growing radially towards the ring area was counted. At least 16 eggs were used for each condition. Means of ratios between vessel number in the test condition and vessel number in the control condition of each animal \pm standard error mean (SEM) were evaluated, and the statistical significance of the differences was determined using the Student's $t$ test (for samples with unequal variance).

\section{Western blot}

AGS or RKO cells, at approximately $80 \%$ confluency, were serum-starved overnight and treated with $\mathrm{CM}$ from unstimulated $(\mathrm{CM}(\mathrm{mac}))$, LPS- (CM(LPS-mac)), IL-10stimulated macrophages (CM(IL-10mac)) or RPMI as control (CMRPMI) for $1 \mathrm{~h}$. Cell lysates, electrophoresis and immunoblotting were performed as previously described [14]. Primary antibodies used included rabbit polyclonal antibodies against phospho-EGFR $\left(\mathrm{Y}^{1086}\right)$ (Zymed-Invitrogen), phospho-Src $\left(\mathrm{Y}^{416}\right)$, phosphoERK1/2( $\left(\mathrm{T}^{202} / \mathrm{Y}^{204}\right)$, phospho-p38 $\left(\mathrm{Thr}^{180} / \mathrm{Tyr}^{182}\right)$, phospho-Akt $\left(\mathrm{S}^{473}\right)$, Akt, ERK1/2, Src, p38 (Cell Signaling, MA, USA), $\alpha$-tubulin (Sigma-Aldrich) or mouse monoclonal antibody against EGFR (Transduction). Donkey antirabbit or sheep anti-mouse-HRP-conjugated secondary antibodies (GE Healthcare) were used, followed by ECLDetection (GE Healthcare).

\section{siRNA transfection}

siRNA targeting EGFR, previously validated for knockdown efficiency in AGS, was purchased from Invitrogen. Prior to transfection, AGS cells at $60 \%$ confluence were incubated in serum-antibiotic-free RPMI1640. Cells were transiently transfected (75 nM EGFR siRNA) using Lipofectamine2000 transfection reagent (Invitrogen). As negative control, cells transfected with Lipofectamine2000 were used. Eight hours after transfection, medium was replaced by RPMI1640 supplemented with $10 \%$ FBS. Knockdown efficiency was tested by western blot, $48 \mathrm{~h}$ after transfection.

\section{Statistical analysis}

Data were analysed using GraphPad Prism v.5 software, and expressed as mean values of at least three independent experiments and \pm Standard Deviations (SD) or Standard Error Mean (SEM), as indicated. Differences were tested with
Mann-Whitney test or Student's $t$ test for non-parametric data, and were considered significant at a $p$ value of less than 0.05. Experiments were performed with at least three different blood donors, as indicated.

\section{Results \\ LPS- and IL-10-stimulated macrophages present distinct phenotypes}

To study the role of distinct macrophage populations on the modulation of cancer cell-related activities, primary human monocytes were first differentiated into macrophages, and left unstimulated or stimulated with $10 \mathrm{ng} / \mathrm{ml}$ of LPS (LPS-stimulated) or IL-10 (IL-10-stimulated), respectively. Macrophage polarization into distinct populations was confirmed by morphology, actin-tubulin cytoskeleton organization, cell surface receptors and cytokine secretion analysis. F-actin and $\alpha$-tubulin staining evidenced differences in cytoskeleton organization between LPS- and IL-10-stimulated macrophages (Fig. 1a). LPSstimulated macrophages were elongated, presenting a significantly higher cell aspect ratio (quotient between cell major and minor axes length) (4.02 \pm 0.03$)$ (Fig. 1b), in comparison with IL-10-stimulated $(1.70 \pm 0.23)$ or with unstimulated macrophages $(2.32 \pm 0.54)$. Unstimulated macrophages constituted a more morphologically heterogeneous population than those stimulated with LPS, which contained areas of pronounced actin staining along the cell body (Fig. 1a, arrows), or those stimulated with IL-10, which contained podosome-like actin protrusions displayed along the entire cell periphery (Fig 1a and b).

The macrophage cytokine profile, performed by ELISA, revealed that LPS-stimulated macrophages produced significantly higher levels of IL- 6 and TNF- $\alpha$, in comparison with IL-10-stimulated ones, which in turn, secreted significantly higher IL-10 and lower IL- 6 and TNF- $\alpha$ levels (Fig. 1c). Flow cytometry analysis of macrophage surface receptors revealed that $\mathrm{CD} 14$, a macrophage lineage marker, was not statistically differently expressed between all macrophage populations (Fig. 1d, left panel). As expected, LPS-stimulated macrophages presented significantly higher expression of the M1-like marker HLA-DR and lower of the M2-like marker CD163 (Fig. 1d, middle panel), in contrast to IL-10-stimulated macrophages, which exhibited significantly lower expression of HLA-DR and higher of CD163 (Fig. 1d, right panel).

Our results are in accordance with those previously described in the literature concerning cytokine and cell surface receptor expression profiles of M1- and M2-like macrophages [29-31].

\section{IL-10-stimulated macrophages are more efficient in stimulating gastric and colorectal cancer cell invasion} To evaluate the influence of distinct macrophage populations on gastric and colorectal cancer cell invasion, 

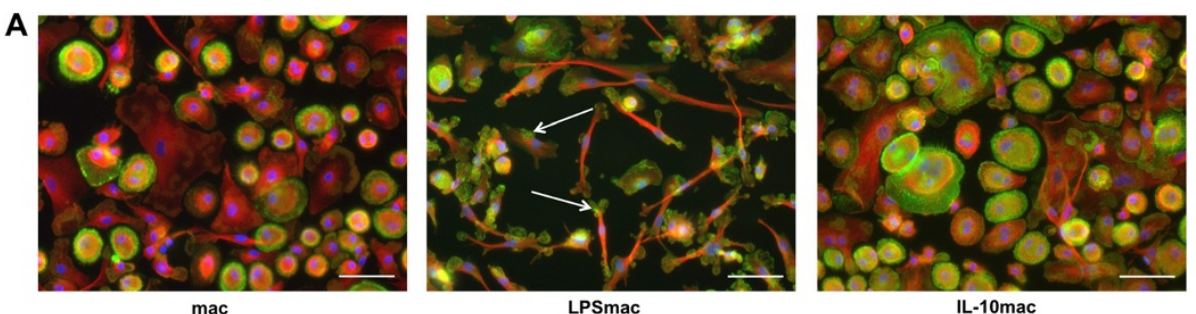

B

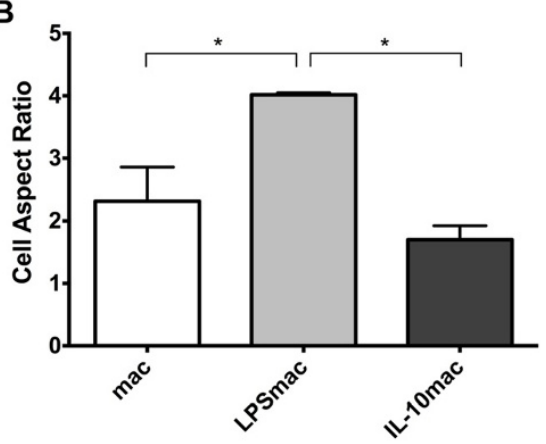

C

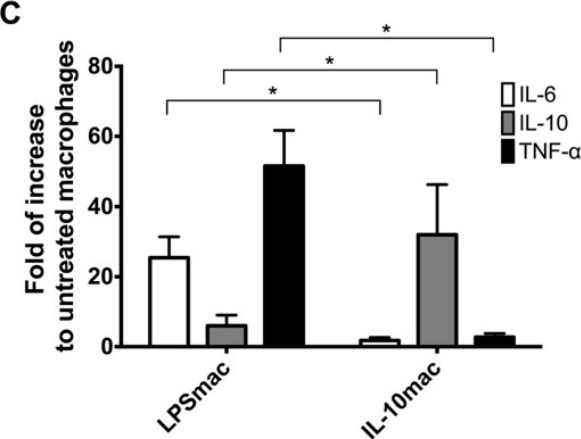

\section{D}

CD14

HLA-DR

CD163
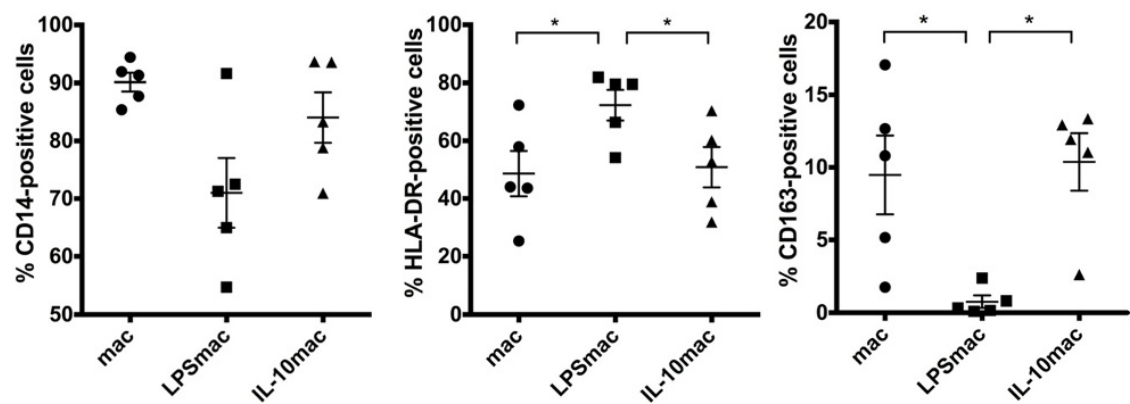

Fig. 1 Phenotypic characterization of LPS- and IL-10-stimulated macrophages derived from human CD14 $4^{+}$peripheral blood monocytes. a Representative images of actin and tubulin stainings of LPS- and IL-10-stimulated macrophages polarized in absence of other external stimuli (mac) or in the presence of 10ng/ml LPS (LPSmac) or IL-10 (IL-10mac), respectively. F-actin was stained with Phalloidin-FITC (green), a-tubulin with a specific monoclonal antibody followed by incubation with AlexaFluor594 secondary antibody (red) and nuclei were counterstained with DAPI (blue). Scale bars represent 50 um. b Morphological differences between macrophage populations were quantified by calculating the cell aspect ratio (quotient between cell major and minor axes) of actin/tubulin stained cells. Chart reflects measurements of at least 100 cells per donor from, at least, 3 distinct donors. Bars represent mean values and flags indicate standard deviations. c Cytokine production profile of LPS- and IL-10-stimulated macrophages. Cytokine concentration was measured by ELISA in conditioned media from distinct macrophage populations. Charts indicate fold increase in IL-6, IL-10 and TNF-a expression, in comparison to unstimulated macrophages. Data is representative of the cytokine profile of cells derived from at least 7 different donors. Bars represent mean values and flags indicate standard deviations. d Expression of typical macrophage lineage (CD14) and polarization markers (HLA-DR and CD163) was determined by flow cytometry of unstimulated, LPS- and IL-10-stimulated macrophages. Scatter charts represent percentage of positive cells for each cell surface marker considering data obtained with cells derived from 5 different donors. ${ }^{*}$, significantly different at $p<0.05$. IL-10, interleukin-10; LPS, lipopolysaccharide

we performed Matrigel invasion assays confronting noninvasive gastric (AGS) or colorectal cancer (RKO) cells with human macrophages, polarized towards an M1 or an M2like phenotype (Fig. 2a and b). Interestingly, the presence of IL-10-stimulated macrophages significantly increased AGS invasion through Matrigel-coated filters, relatively to AGS cells alone and to LPS-stimulated macrophages. The latter were still effective in inducing AGS cell invasion (fold of increase $4.41 \pm 0.56$ ), although to a significantly lower extent than IL-10-stimulated macrophages $(14.11 \pm 1.89)$ (Fig. 2a).
The observed stimulation of invasion seems to occur by the action of one or more soluble factors since, in this experimental system, the two cell types do not contact directly. Similarly, IL-10-stimulated macrophages induced RKO invasion (fold of increase $2.935 \pm 0.20$ ) in a higher extent than LPS-stimulated macrophages (1.203 \pm 0.24$)$ (Fig. 2b). As controls, $10 \mathrm{ng} / \mathrm{ml} \mathrm{LPS} \mathrm{or} 10 \mathrm{ng} / \mathrm{ml} \mathrm{IL-10}$ alone were added to invasion assays in the absence of macrophages, having no influence on the number of invasive cancer cells (data not shown). 

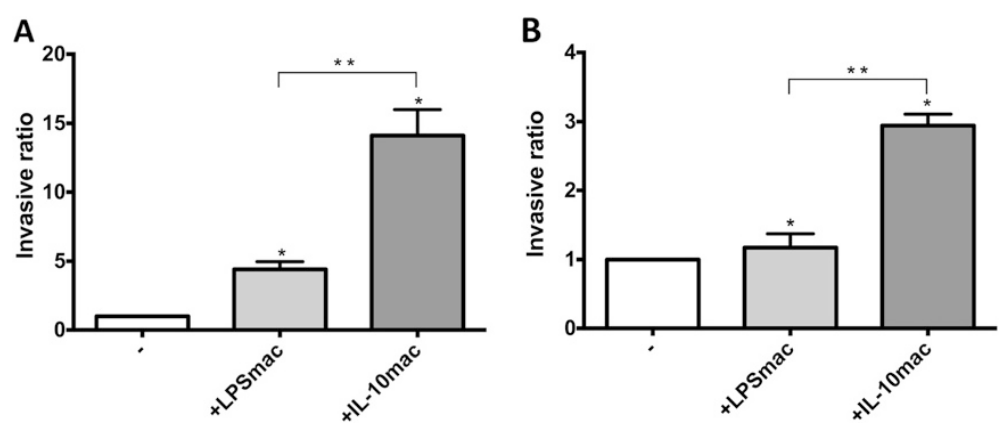

Fig. 2 IL-10-stimulated macrophages are more efficient in stimulating cancer cell invasion. a AGS human gastric or $\mathbf{b}$ RKO human colorectal cancer cells were incubated in BD BioCoat ${ }^{\mathrm{TM}}$ Matrige ${ }^{\mathrm{TM}}$ Invasion Chambers for $24 \mathrm{~h}$ with RPMl medium (-), or human macrophages differentiated for 10 days and stimulated for $72 \mathrm{~h}$ with $10 \mathrm{ng} / \mathrm{ml}$ LPS (LPSmac) or $10 \mathrm{ng} / \mathrm{ml} \mathrm{IL-10} \mathrm{(LL-10mac).} \mathrm{Invasive} \mathrm{cells} \mathrm{and} \mathrm{invasive} \mathrm{ratio} \mathrm{were} \mathrm{determined} \mathrm{as} \mathrm{described} \mathrm{in} \mathrm{Materials}$ and Methods. Bars represent mean values of independent experiments performed with, at least, 4 different donors; flags indicate standard deviations

Altogether, these results indicate that IL-10- and LPSstimulated macrophages affect gastric and colorectal cancer cell invasion in different extents, being the IL-10stimulated more efficient. Since AGS cells were more susceptible to macrophage-mediated invasion than RKO, as indicated by the invasive ratios obtained, these cells were selected for subsequent studies.

\section{IL-10-stimulated macrophages are more efficient in inducing cancer cell motility and migration}

To investigate the effect of IL-10- and LPS-stimulated macrophages on cancer cell motility and cytoskeleton organization, F-actin and $\alpha$-tubulin stainings were conducted on AGS cells, after 6 h-treatments with CM from both macrophage populations or with control RPMI medium. In response to soluble factors produced by both IL-10- and LPS-stimulated macrophages, motility-associated structures, such as filopodia and lamellipodia, were observed (Fig. 3a, arrows). These actin-rich structures, essentially filopodia, were more frequent on cells exposed to CM from IL-10-stimulated $(\mathrm{CM}(\mathrm{IL}-10 \mathrm{mac}))(38.7 \pm 5.2 \%)$, than on cells exposed to CM from LPS-stimulated macrophages (CM(LPSmac)) $(17.3 \pm 2.5 \%)$ or to control RPMI $(9.9 \pm 1.7 \%)$ (Fig. 3b). In addition, cells exposed to CM from IL-10-stimulated macrophages had a more elongated shape, than cells exposed to RPMI only (AGS + RPMI). The latter presented a pronounced polyhedral shape and a strong cortical actin staining at the periphery (Fig. 3a). Aiming to study the effect of both macrophage populations on cancer cell migration, high-resolution timelapse microscopy was performed for $13 \mathrm{~h}$. In the presence of CM from IL10-stimulated macrophages, cancer cells described wider trajectories (Fig. 3c) and travelled significantly longer distances (Fig. 3d) in comparison to cancer cells stimulated with CM from LPS-stimulated macrophages $(\mathrm{CM}(\mathrm{LPSmac}))$. Overall these results indicate that IL10-stimulated macrophages are more efficient than
LPS-sitmulated macrophages in inducing cancer cell migration.

\section{IL-10-stimulated macrophages promote cancer cell mediated-angiogenesis}

Angiogenesis is known to be crucial in several steps of tumour progression and M2-like macrophages are described to have pro-angiogenic potential [32]. To explore the influence on new vessels formation of IL-10- and LPSstimulated macrophages, we incubated AGS cells with $\mathrm{CM}$ from both populations (AGS + CM(LPSmac) and AGS + CM(IL-10mac), respectively) in the chick embryo chorioallantoic membrane (Fig. 4a). As internal control, AGS cells with RPMI medium (AGS + RPMI) were included in each egg. Our results show that inoculation of AGS cells with CM from IL-10-stimulated macrophages resulted in a significantly higher number of new vessels, growing outwards the inoculation area (Fig. 4b, bottom chart). This indicates that soluble factors produced by this specific macrophage population are stimulating cancer cell-mediated angiogenesis. Conversely, CM from LPSstimulated macrophages decreased the angiogenic response, since the number of new vessels formed was lower than in the control (Fig. 4b, upper chart). Altogether, our results suggest that molecules produced by IL-10-stimulated macrophages switch the balance of pro- and anti-angiogenic molecules towards the stimulation of angiogenesis, while unstimulated and LPS-stimulated macrophages have the opposite effect.

Cancer cell invasion requires activation of EGFR signalling in the presence of IL-10- and LPS-stimulated macrophages

EGFR and its associated signalling molecules have been previously implicated in macrophage-derived stimuli of invasion. In fact, silencing cancer cell EGFR expression or immunodepletion of EGF from macrophage CM, led to inhibition of motility, and abrogation of cancer cell 

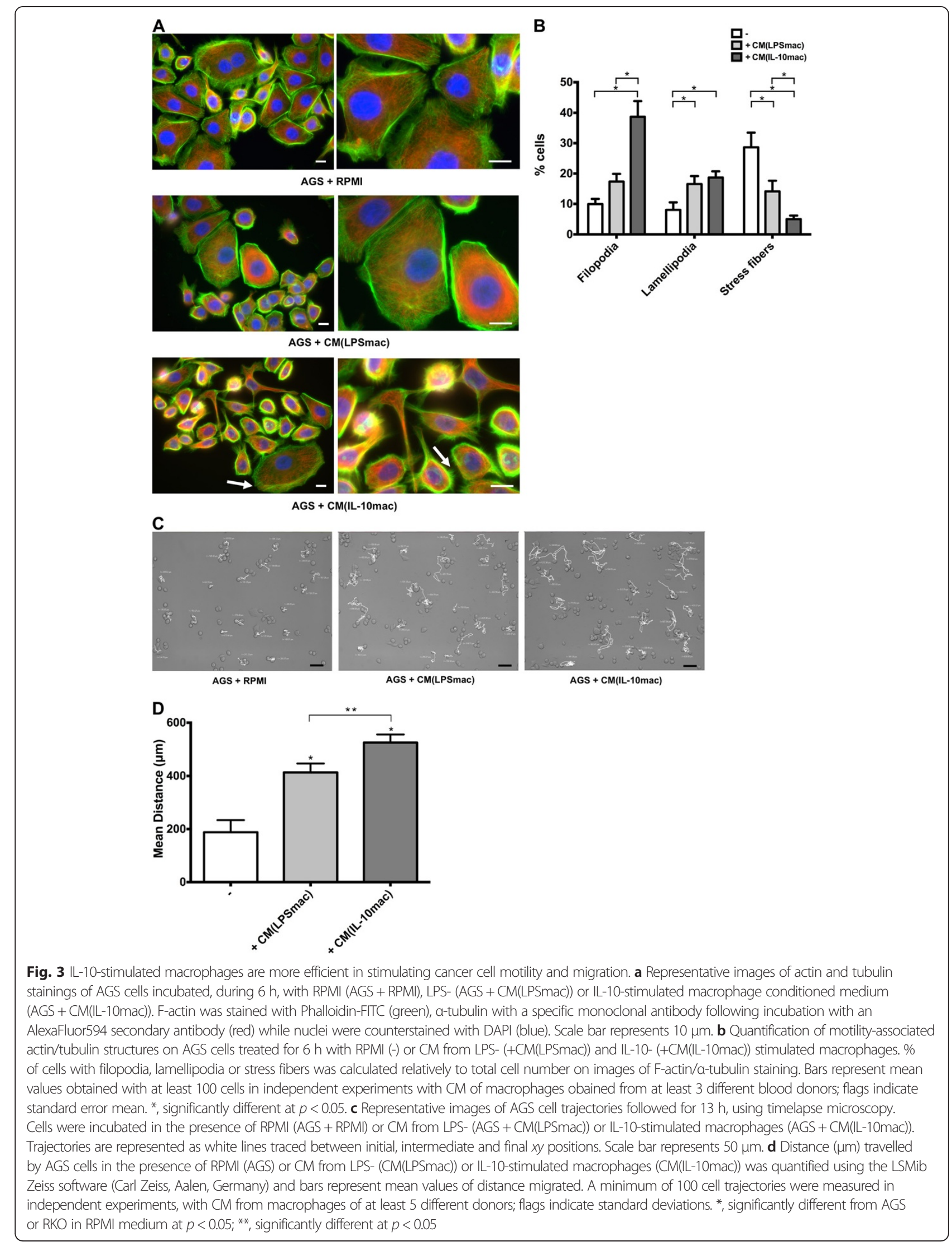
A

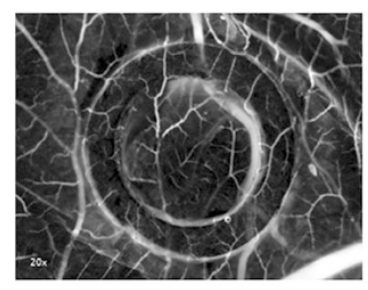

AGS + RPMI

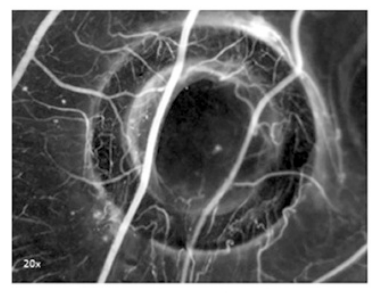

AGS + RPMI

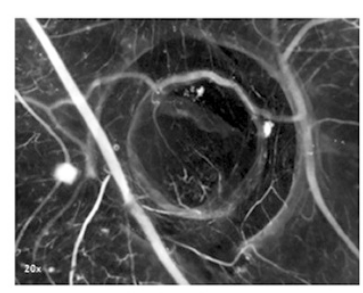

AGS + CM(LPSmac)

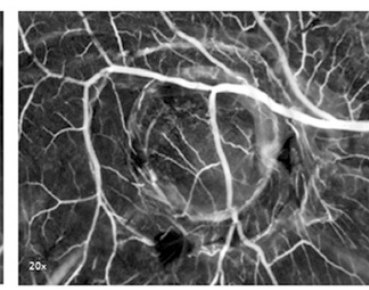

AGS + CM(IL-10mac)
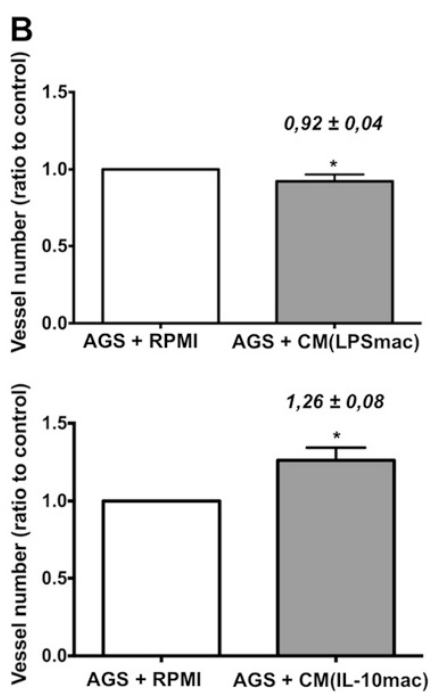

Fig. $4 \mathrm{IL}-10$-stimulated macrophages promote cancer cell angiogenic response in the chick embryo chorioallantoic membrane (CAM) assay. a Representative images of the CAM showing AGS cell inoculation area (ring delimited). A control with AGS and RPMI (AGS + RPMI) was always included in each egg (20 X magnification), next to the inoculation site of AGS with CM from LPS- (AGS + CM(LPSmac)) or IL-10-stimulated $(A G S+C M(I L-10 m a c))$ macrophages. b Quantification of the number of new blood vessels grown towards each inoculation area (only vessels $<20 \mu \mathrm{m}$ diameter were counted). This quantification is compared with the control condition (AGS + RPMI) present at each egg (ratio between the vessel number in the test condition and the vessel number in the control condition). Bars represent mean values obtained with 18 eggs for AGS + CM(LPSmac), and 16 eggs for AGS + CM(IL-10mac) and flags indicate standard error mean *, significantly different at $p<0.05$

invasion [14]. Therefore, to evaluate if EGFR is also necessary for IL-10- and LPS-stimulated macrophage-mediated invasion, AGS cells transiently silenced with siRNA for this receptor were confronted with both macrophage populations on Matrigel invasion assays. Since silencing of EGFR reached its maximum $48 \mathrm{~h}$ post-transfection (Fig. 5a), invasion assays were performed $24 \mathrm{~h}$ after transfection. A significant decrease in the invasion ability of cancer cells confronted with IL-10-stimulated macrophages was observed upon EGFR silencing, even when comparing with AGS cells transfected with Lipofectamine only. The same inhibitory effect was observed for LPS-stimulated macrophages, although not significantly, probably due to the lower levels of invasion already induced by this macrophage population (Fig. 5a).

Taking into account that EGFR is required for IL-10- and LPS-stimulated macrophage-mediated invasion, we evaluated if both macrophage populations differently affected cancer cell EGFR signalling. Interestingly, no differences in terms of EGFR tyrosine phosphorylation were found among AGS cells treated with CM from these distinct macrophage populations (Fig. 5b). Comparable patterns of intense membrane phosphoEGFR immunostaining were observed, whereas in the control condition (AGS + RPMI), the levels of phosphorylation were low and scattered throughout the cytoplasm. To evaluate the effect of distinct macrophage populations on the activation of EGFR signalling partners, lysates of AGS or RKO cells, previously incubated or not with CM from unstimulated (CMmac), LPS- (CM(LPSmac)) and IL-10-stimulated macrophages (CM(IL-10mac)), were analysed by immunoblotting. Our results indicate that, after $1 \mathrm{~h}$, the three macrophage populations induce similar increases in the phosphorylation of EGFR $\left(\Upsilon^{1086}\right)$, c-Src $\left(\Upsilon^{416}\right)$, ERK1/2 $\left(\mathrm{T}^{202} / \Upsilon^{204}\right)$, AKT $\left(\mathrm{S}^{473}\right)$ and $\mathrm{p} 38\left(\mathrm{~T}^{180} /\right.$ $\mathrm{T}^{182}$ ) (Fig. 5c, left panel) in AGS cells. Since no differences in activation were observed in gastric cancer cells, similar studies were conducted with colorectal cancer cells. Similar results were obtained in terms of RKO cells phosphorylation of EGFR $\left(\Upsilon^{1086}\right)$, c-Src $\left(\Upsilon^{416}\right)$ and AKT $\left(S^{473}\right)$, upon treatments with CM from unstimulated, LPS- and IL-10-stimulated macrophages (Fig. 5c, right panel). Altogether, these results point EGFR signalling as fundamental for the induction of invasion provided by IL-10and by LPS-stimulated-macrophages, as silencing EGFR in AGS cells caused them to be unresponsive to stimulation of invasion from both macrophage subsets. This effect is more prominent in terms of IL-10-treated macrophages stimulation because LPS-treated macrophages already cause lower levels of AGS invasion even with intact EGFR expression. These observations led us to the conclusion that other factors might be responsible for the distinct pro-invasive ability of both macrophage populations.

\section{Macrophage-mediated invasion and angiogenesis are dependent on MMP activity}

Proteolysis is a critical event in the progression of cancer [33-35] and we previously reported that macrophages are the major contributors to the enhanced proteolysis 

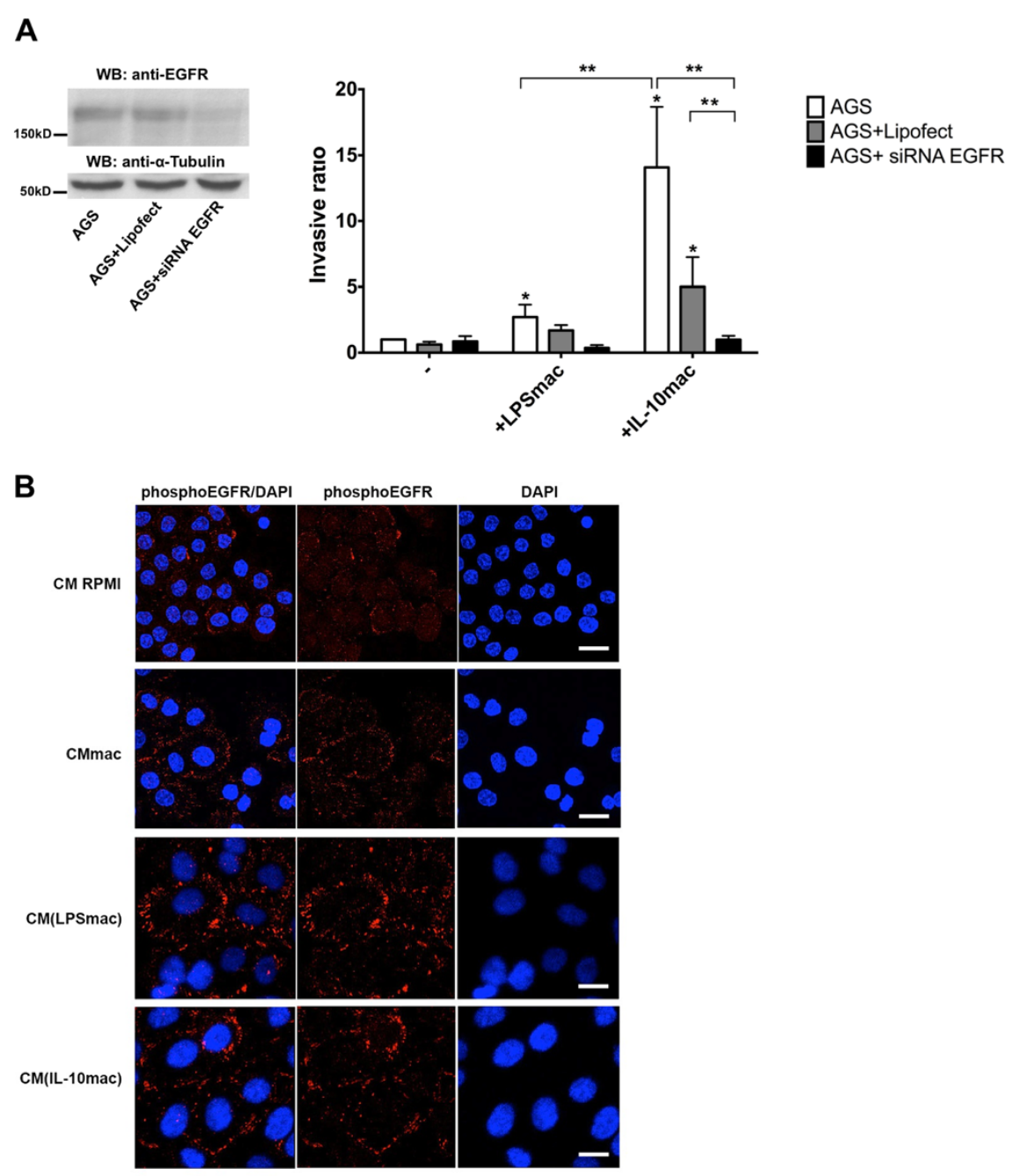

C

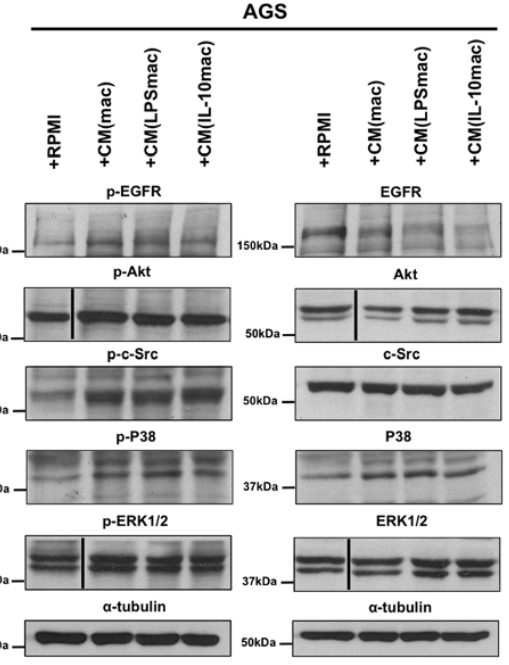

RKo

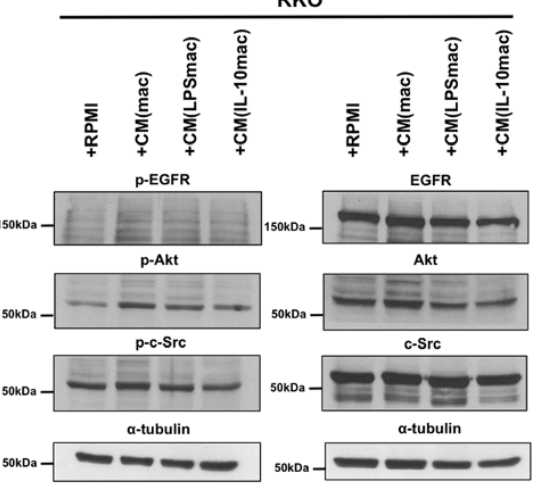

Fig. 5 (See legend on next page.) 
(See figure on previous page.)

Fig. 5 EGFR is required for both LPS- and IL-10-stimulated macrophage-mediated gastric cancer cell invasion. a EGFR expression was transiently silenced by transfection with validated siRNA, at $48 \mathrm{~h}$ post-transfection. b Invasion assays with AGS cells (AGS), AGS cells with Lipofetamine2000 (AGS + Lipofect) or with Lipofectamine2000 transfected together with siRNA directed to EGFR (AGS + siRNA EGFR) were performed in the presence or absence of LPS- (LPSmac) or IL-10-stimulated (IL-10 mac) macrophages. These assays were conducted at $24 \mathrm{~h}$ post-transfection and stopped at $48 \mathrm{~h}$ post-transfection, when maximum inhibition was achieved. Bars represent mean values of independent experiments performed with macrophages from at least 4 different donors and flags indicate standard error mean * significantly different from AGS at $p<0.05$; * significantly different at $p<0.05$. c Tyrosine phosphorylation status of EGFR residue Y $^{1086}$ (red) after $1 \mathrm{~h}$ of incubation of AGS cells with RPMI (CM RPMI) or CM from unstimulated (CMmac), LPS- (CM(LPSmac)) or IL-10-stimulated (CM(IL-10mac)) macrophages. Nuclei were counterstained with DAPI (blue). Scale bar represents $10 \mu \mathrm{m}$. The image is representative of independent experiments performed with CM of macrophages, from at least three different donors. $\mathbf{d}$ AGS or RKO cells were treated or not (RPMI), during $1 \mathrm{~h}$, with CM from unstimulated (CMmac), LPS- (CM(LPSmac)) or IL-10-stimulated (CM(IL-10)mac) macrophages. Cell lysates were immunoblotted for phosphorylated and total EGFR $\left(Y^{1086}\right), c-S r c\left(Y^{416}\right)$, Akt $\left(S^{473}\right)$, ERK1/2 $\left(T^{202} \mathrm{Y}^{204}\right)$, and $\mathrm{p38}\left(\mathrm{Thr}{ }^{180} / \mathrm{Tyr}^{182}\right)$. Immunoblots were analyzed by densitometry analysis in comparison with corresponding a-tubulin and total protein expression levels. Images are representative of independent experiments performed with CM of macrophages from at least 3 different donors

found in co-cultures with cancer cells. Moreover we showed that, besides EGFR, matrix metalloproteases (MMP) were crucial for macrophage-mediated cancer cell invasion, since the presence of a broad MMP inhibitor (Galardin) was able to abolish this effect [14]. Thus, invasion assays with AGS, in the presence or absence of LPS - (CM(LPSmac)) or IL-10-stimulated (CM(IL-10mac)) macrophages, were carried out in the presence of Galardin (Fig. 6a). In fact, by inhibiting MMPs, both LPS- and IL10-stimulated macrophage-mediated stimuli of invasion were significantly reduced.

Since MMP activity is important in terms of angiogenesis [36] and that CM from IL-10-stimulated macrophages increased the angiogenic response in the chick embryo CAM assay, we inoculated AGS cells with CM from IL-10stimulated macrophages with Galardin (AGS + CM(IL-10mac) + Galardin), using a control condition, in the same egg, without the inhibitor (AGS + CM(IL-10-mac)) (Fig. 6b). The presence of Galardin resulted in a slight, although statistically significant, decrease in the ability of IL-10-stimulated macrophages to induce cancer cell-mediated angiogenesis. These results indicate that MMPs are required to support cancer cell invasion provided by both macrophage populations, and to the stimulation of angiogenesis induced by CM derived from IL-10-stimulated macrophages.

\section{IL-10-stimulated macrophages display enhanced MMP-2 and MMP-9 activities, particularly in the presence of cancer cells}

Considering the ability of a broad-MMP inhibitor to decrease cancer cell invasion and cancer cell-induced angiogenesis, we hypothesized that the distinct ability of LPS- and IL-10-stimulated macrophages in inducing these cancer cell activities could be associated to proteolytic differences. Therefore, gelatin zymography studies were conducted using $\mathrm{CM}$ from both macrophage-populations, when cultured alone or with cancer cells (Fig. 6c). Our results show that IL-10-stimulated macrophages (CMMat(IL-10mac)) present significantly higher pro- and active-MMP-9 activities than LPS-stimulated macrophages (CMMat(LPSmac)) (Fig. 6d).
Concerning AGS cells (CMMat(AGS)), levels of MMP-9 are similar to those of LPS-stimulated but significantly lower from those of IL-10-stimulated macrophages. Regarding the proteolytic activity of invasion assays supernatants, pro- and active-MMP-9 levels in co-cultures of AGS cells with LPS-stimulated macrophages (CMMa$\mathrm{t}(\mathrm{AGS}+\mathrm{LPS}$ mac) are comparable to individual population levels. Nevertheless, pro- and active-MMP-9 proteolytic activity is significantly higher in co-cultures of AGS with IL-10(CMMat(AGS+IL-10mac)) than with LPS-stimulated macrophages (CMMat(AGS + LPSmac)) (Fig. 6d). Regarding MMP-2, both pro- and active forms were significantly higher in IL-10- than in LPS-stimulated macrophages (Fig. 6e). Considering co-cultures, pro- and active-MMP-2 activity was considerably higher in AGS cells with IL-10stimulated (CMMat(AGS + IL-10mac)) than in the corresponding condition with LPS-stimulated macrophages (CMMat(AGS + LPSmac)). In fact, no differences on MMP-2 activity were observed between LPS-stimulated macrophages alone and in co-cultures with AGS cells (Fig. 6e), suggesting that this metalloprotease is mainly produced by macrophages.

Taken together, these results indicate that differences in the stimuli of invasion provided by LPS- and IL-10stimulated macrophages are probably related with distinct proteolytic activity profiles. Besides similar abilities in stimulating EGFR phosphorylation, IL-10-stimulated macrophages provide, in fact, higher MMP-2 and MMP9 activities than their LPS-stimulated counterparts.

\section{Discussion}

In this study, we investigated the role of M1 versus M2like macrophages on gastric and colorectal cancer cell functions. Our results demonstrate that: 1) macrophages distinctly modulate cancer cell invasion, motility, proteolysis and angiogenesis, being M2-like macrophages (IL-10stimulated) more efficient than their M1-like counterparts (LPS-stimulated); 2) EGFR phosphorylation is essential for both macrophage populations-mediated invasion; 3) despite differences in their pro-invasive ability, LPS- and IL- 

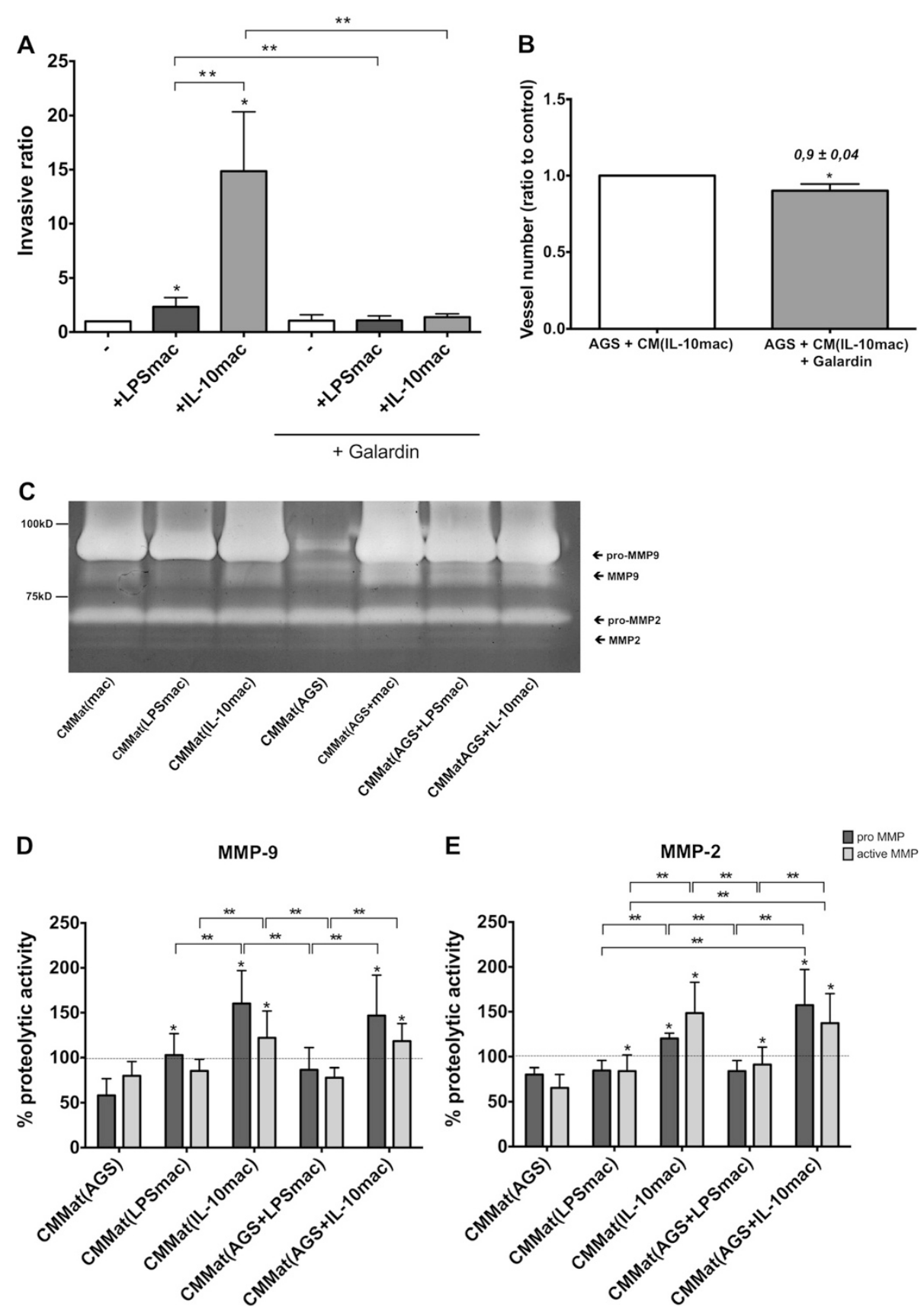

Fig. 6 (See legend on next page.) 
(See figure on previous page.)

Fig. 6 MMP activity influences LPS- and IL-10-stimulated macrophage-mediated cancer cell invasion and angiogenesis. a AGS cells were incubated in BD BioCoat ${ }^{\mathrm{TM}}$ Matrigel ${ }^{\mathrm{TM}}$ Invasion Chambers for $24 \mathrm{~h}$ with RPMl medium (-) or macrophages stimulated for $72 \mathrm{~h}$ with $10 \mathrm{ng} / \mathrm{ml} \mathrm{LPS}$ (LPSmac)) or with $10 \mathrm{ng} / \mathrm{ml}$ IL-10 (IL-10mac) and supplied or not with a pharmacological inhibitor of matrix metalloproteases, Galardin (10 $\mu \mathrm{M})$. Invasive cells were determined as described in Materials and Methods. Bars represent mean values of independent experiments performed with, at least, 4 different donors; flags indicate standard deviations. *, significantly different from AGS in RPMI medium at $p<0.05$; ${ }^{* *}$, significantly different at $p<0.05$. $\mathbf{b}$ Quantification of the number of new blood vessels grown in the CAM towards each inoculation area ( $<20 \mu \mathrm{m}$ diameter). A control with AGS and CM from IL-10-treated macrophages without Galardin $(A G S+C M((L-10 \mathrm{mac}))$ was always included in each egg next to the inoculation area of AGS and CM of IL-10-stimulated macrophages supplemented with Galardin $(30 \mu \mathrm{M})(\mathrm{AGS}+\mathrm{CM}(\mathrm{IL}-10 \mathrm{mac})+$ Galardin). Bars represent mean values (ratio between the vessel number in the test condition and the vessel number in the control condition, per animal) obtained from a total of 35 eggs and CM of macrophages derived from 3 different donors. Flags indicate standard error mean; *, significantly different at $p<0.05$. c Conditioned media from Matrigel ${ }^{\mathrm{TM}}$ invasion assays containing AGS (CMMat(AGS)), unstimulated (CMMat(mac)), LPS- (CMMat(LPSmac)), L-10-stimulated macrophages (CMMat(IL-10mac)), AGS and unstimulated (CMMat(mac)), LPS- (CMMat(AGS + LPSmac)) and IL-10stimulated macrophages (CMMat(AGS + L-10mac)) were run on gelatin zymograms. Proteolytic activity bands were revealed in white on a blue background stained with Coomassie. $\mathbf{d}$ and e Densitometry analysis using QuantityOne ${ }^{\oplus}$ software (BioRad) allowed quantification of pro-MMP-9 and MMP-9 (d) and pro-MMP-2 and MMP-2 (e) activities. Proteolytic activity was expressed as percentage of the proteolytic activity of unstimulated macrophages. Data correspond to mean values of independent experiments performed with macrophages derived from at least 5 different blood donors. Flags indicate standard error mean; *, significantly different from AGS at $p<0.05$; ${ }^{*}$, significantly different at $p<0.05$

10-stimulated macrophages similarly stimulate cancer cell EGFR, c-Src, ERK1/2, Akt and p38 phosphorylation; 4) most importantly, MMPs are crucial for macrophagemediated invasion and angiogenesis and discrepancies in the strength of these stimuli seems related with differences in the MMP activity profile of each macrophage population. As such, the higher proteolytic activity of IL-10stimulated macrophages parallels with their higher ability to stimulate cancer cell invasion and angiogenesis.

Due to their pro-inflammatory phenotype, M1 macrophages are often associated with tumour-suppressor and cytotoxic activities. In contrast, M2-like macrophages, which display an anti-inflammatory phenotype, are generally engaged in tumour-promoting activities, in particular angiogenesis and extracellular matrix remodelling [19]. These macrophage populations also exhibit distinct molecular profiles, which are, to a certain extent, associated with the microenvironment to where monocytes are recruited and differentiated [4]. In our system, these two distinct macrophage phenotypes were obtained upon exogenous stimulation of primary human monocytes, and exhibited previously described characteristics of extremes in an activation spectrum [22]. M2-like macrophages, which share characteristics of tumour-associated macrophages (TAMs) [19], were indeed more efficient in stimulating gastric and colorectal cancer cell invasion than their LPS-stimulated counterparts. This finding is in agreement with the ability of M2like macrophages to stimulate cancer cell migration, invasion and metastasis in other types of tumours [1]. Interestingly, LPS-stimulated macrophages also increased cancer cell invasion, although to a less extent. Accordingly, Hagemann and collaborators have previously described that LPSstimulated macrophages were less efficient in promoting breast cancer cell invasion than naïve macrophages [37].

Cancer cell invasion results from the balance between migration and proteolysis, which facilitates the movement of cells through extracellular matrix (ECM) degraded components, allowing the release of pro-invasive and pro- motility factors entrapped within the matrix $[33,35,38]$. We have previously demonstrated that cancer cell motility is another crucial invasion-related activity stimulated by naïve macrophages [14]. In the present study, LPS- and IL-10stimulated macrophages induced cancer cell cytoskeleton reorganization and the formation of motility-associated structures, such as lamellipodia and filopodia. Nevertheless, soluble molecules produced by IL-10-stimulated macrophages were significantly more efficient in inducing cancer cell migration than those released by LPS-stimulated macrophages. A paracrine loop involving the production of EGF by macrophages and CSF-1 by cancer cells has been demonstrated [13]. Disruption of this cellular crosstalk by blockade of EGF receptor or CSF-1 receptor signalling inhibited cancer cell migration and invasion [13]. We have also recently described the relevance of EGF-like ligands, produced by naïve macrophages, for the activation of gastric and colorectal cancer cell. EGFR and its downstream partners such as Akt, ERK1/2 and c-Src were proved to be crucial for stimulation of invasion and motility [14]. Interestingly, in the present work we demonstrate that these signalling pathways are being similarly induced by LPS- and IL-10-stimulated macrophages, despite their distinct effects on cancer cell invasion and motility. In fact, soluble factors released by both macrophage populations induced similarly EGFR, c-Src, ERK1/2, Akt, and p38 phosphorylation. Thus, our results indicate that, although critical, this pathway is not responsible for the differences in invasion-related cellular activities promoted by LPS- and IL-10-stimulated macrophages, pointing to a distinct mechanism.

MMPs are essential for tumour cell invasion through the basement membrane $[35,38]$ and increased expression has been positively correlated with tumour progression $[36,39]$. Host cells, especially macrophages, are considered the main source of these enzymes at the tumour microenvironment [40] and we and others have recently reported that macrophage-mediated cancer cell invasion and migration are sensitive to MMP inhibition [14, 37]. In 
the present study, the higher MMP activity exerted by IL-10-stimulated macrophages seems to be associated with the enhancement of gastric cancer cell invasion. As previously, proteolytic activity inhibition impaired macrophagemediated invasion [14, 37]. Taken together, our results suggest that M2-like macrophages are more efficient than their M1 counterparts in stimulating gastric cancer cell invasion, likely due to their increased MMP-2 and MMP-9 activity and higher ability to promote cancer cell migration. With increased capacity to degrade ECM components and enhanced motility and migration, the invasive ability of gastric cancer cells might be then supported.

Tumour-induced angiogenesis is essential for tumour progression since it is important for local tumour growth, survival, escape from the primary site and establishment of metastasis [41]. Macrophages are described as producers of pro-angiogenic cytokines and growth factors [42] and have already been associated with tumours neovascularization [43-45]. Nevertheless, different angiogenic potentials have been associated with distinct macrophage populations, with M2-like macrophages presenting proangiogenic features $[46,47]$. Consistent with this, we observed stimulation of gastric cancer cell-induced angiogenesis in vivo by soluble factors secreted by M2-like macrophages. Since MMPs are crucial regulators at distinct steps of the angiogenic process $[36,48]$, we propose that the angiogenic stimulatory effect of M2 macrophages could be related to their enhanced MMP-2 and MMP-9 activities. However, we cannot exclude that other proangiogenic factors, secreted by this macrophage population might also be involved. M1 macrophages, on the other hand, were previously reported to inhibit angiogenesis in mice, even in the presence of increased levels of vascular endothelial growth factor (VEGF) [47].

\section{Conclusions}

Overall, our results provide valuable insights into the interplay between cancer cells and two distinctly polarized macrophage populations, frequently found at the tumour microenvironment. By examining the molecular mechanisms and highlighting the importance of MMPs in such cellular crosstalk, this work offers new considerations to the establishment of more efficient therapeutic strategies, aiming to impair cancer invasion and progression.

\section{Competing interests}

The authors declare that they have no competing interests.

\section{Authors' contributions}

APC designed the study, performed the experiments and drafted the manuscript. MLP and ATP carried out in vitro and in vivo CAM assay experiments. CM performed in vitro experiments. MIO and SGS planned the study and performed in vitro experiments. JBR, RS, MM an MAB supervised the data and drafted the manuscript. MJO designed the study, supervised the data and drafted the manuscript. All authors read and approved the final manuscript.

\section{Acknowledgements}

This work was financially supported by Programa Operacional Factores de Competitividade-COMPETE and through FCT-Portuguese Science Foundation in the famework of projects PEst-C/SAU/LA0002/2013 and UID/BIM/04293/2013, FCT(PTDC-SAU-ONC/112511/2009) and Prize L'Oreal for Women in Science (Foundation L'Óreal/FCT/UNESCO). APCardoso is a PhDFellow from the Iberian Nanotechnology Laboratory (INL). ATPinto and MLPinto are PhDFellows (SFRH/ BD/74144/2010, SFRH/BD/81103/2011) and MIOliveira a PostDocFellow (SFRH/ BPD/37090/2007) from FCT. MTPinto was granted by FCT-Program Ciência2008. SGSantos was funded by North Region Operational Program (ON.2), project NORTE-07-0124-FEDER-000005-QREN. MJ Oliveira is an InvestigatorFCT recipient.

\section{Author details}

${ }^{1}$ i3S-Instituto de Investigação e Inovação em Saúde/INEB-Institute of Biomedical Engineering, University of Porto, Porto, Portugal. ${ }^{2}$ FEUP-Faculty of Engineering, University of Porto, Porto, Portugal. ${ }^{3}$ ICBAS-Institute of Biomedical Sciences Abel Salazar, University of Porto, Porto, Portugal. ${ }^{4}$ i3S-Instituto de Investigação e Inovação em Saúde/IPATIMUP-Institute of Molecular Pathology and Immunology of the University of Porto, Porto, Portugal. ${ }^{5}$ i3S-Instituto de Investigação e Inovação em Saúde/IBMC-Institute for Cell and Molecular Biology, University of Porto, Porto, Portugal. ${ }^{6}$ Department of Pathology and Oncology, Faculty of Medicine, University of Porto, Porto, Portugal. 'Humanitas Clinical and Research Centre, Rozzano, Italy. ${ }^{8}$ BIOMETRA Department, University of Milan, Milan, Italy. ${ }^{9}$ Laboratory of Experimental Cancerology, Ghent University Hospital, Ghent, Belgium.

Received: 12 October 2014 Accepted: 21 May 2015

Published online: 05 June 2015

\section{References}

1. Condeelis J, Pollard JW. Macrophages: obligate partners for tumor cell migration, invasion, and metastasis. Cell. 2006;124(2):263-6.

2. Bingle $L$, Brown $N$, Lewis $C$. The role of tumour-associated macrophages in tumour progression: implications for new anticancer therapies. J Pathol. 2002;196(3):254-65.

3. Qian BZ, Pollard JW. Macrophage diversity enhances tumor progression and metastasis. Cell. 2010:141(1):39-51.

4. Mantovani A, Bottazzi B, Colotta F, Sozzani S, Ruco L. The origin and function of tumor-associated macrophages. Immunol Today. 1992;13(7):265-70.

5. Zhang QW, Liu L, Gong CY, Shi HS, Zeng YH, Wang XZ, et al. Prognostic significance of tumor-associated macrophages in solid tumor: a meta-analysis of the literature. PLoS ONE. 2012;7(12), e50946.

6. Edin S, Wikberg ML, Dahlin AM, Rutegård J, Öberg Å, Oldenborg P-A, Palmqvist R. The distribution of macrophages with a M1 or M2 phenotype in relation to prognosis and the molecular characteristics of colorectal cancer. PLOS ONE. 2012;7(10):e47045.

7. Zlobec I, Lugli A. Invasive front of colorectal cancer: dynamic interface of pro-/anti-tumor factors. World J Gastroenterol. 2009;21 (15(47)):5898-906.

8. Galon J, Costes A, Sanchez-Cabo F, Kirilovsky A, Mlecnik B, Lagorce-Pages C, et al. Type, density, and location of immune cells within human colorectal tumors predict clinical outcome. Science. 2006;313(5795):1960-4.

9. Ohno S, Inagawa H, Dhar D, Fujii T, Ueda S, Tachibana M, et al. The degree of macrophage infiltration into the cancer cell nest is a significant predictor of survival in gastric cancer patients. Anticancer Res. 2003;23(6D):5015-22.

10. Ong S-M, Tan Y-C, Beretta O, Jiang D, Yeap W-H, Tai JJY, et al. Macrophages in human colorectal cancer are pro-inflammatory and prime T cells towards an anti-tumour type-1 inflammatory response. Eur J Immunol. 2012;42(1):89-100.

11. Ishigami S, Natsugoe S, Tokuda K, Nakajo A, Okumura H, Matsumoto M, et al. Tumor-associated macrophage (TAM) infiltration in gastric cancer. Anticancer Res. 2003;23(5A):4079-83.

12. Shen Z, Kauttu T, Seppänen H, Vainionpää S, Ye Y, Wang S, et al. Both macrophages and hypoxia play critical role in regulating invasion of gastric cancer in vitro. Acta Oncol. 2013;52(4):852-60.

13. Goswami S, Sahai E, Wyckoff JB, Cammer M, Cox D, Pixley FJ, et al. Macrophages promote the invasion of breast carcinoma cells via a colony-stimulating factor-1/ epidermal growth factor paracrine loop. Cancer Res. 2005;65(12):5278-83.

14. Cardoso AP, Pinto ML, Pinto AT, Oliveira MI, Pinto MT, Gonçalves RM, et al. Macrophages stimulate gastric and colorectal cancer invasion through EGFR Y1086, c-Src, Erk1/2 and Akt phosphorylation and smallGTPase activity. Oncogene. 2013;33(16):2123-33. 
15. Mantovani A, Allavena P, Sozzani S, Vecchi A, Locati M, Sica A. Chemokines in the recruitment and shaping of the leukocyte infiltrate of tumors. Semin Cancer Biol. 2004;14(3):155-60.

16. Cortez-Retamozo V, Etzrodt M, Newton A, Rauch PJ, Chudnovskiy A, Berger $C$, et al. Origins of tumor-associated macrophages and neutrophils. Proc Natl Acad Sci. 2012;109(7):2491-6.

17. Mukaida N, Baba T. Chemokines in tumor development and progression. Exp Cell Res. 2012;318(2):95-102.

18. Cassetta L, Cassol E, Poli G. Macrophage polarization in health and disease. Sci World J. 2011;11:2391-402.

19. Mantovani A. Macrophage polarization: tumor-associated macrophages as a paradigm for polarized M2 mononuclear phagocytes. Trends Immunol. 2002;23(11):549-55.

20. Orlikowsky T, Dannecker G, Wang Z, Horowitz H, Niethammer D, Hoffmann M. Activation or destruction of T cells via macrophages. Pathobiology. 1999:67:298-301.

21. Hamilton TA. Molecular basis of macrophage activation: from gene expression to phenotypic diversity. In: Bourke BaL C, editor. The macrophage. Oxford: Oxford University Press; 2002. p. 73-102.

22. Mantovani A. The chemokine system in diverse forms of macrophage activation and polarization. Trends Immunol. 2004;25(12):677-86.

23. Talmadge JE. Inflammatory cell infiltration of tumors: Jekyll or Hyde. Cancer Metastasis Rev. 2007;26:373-400.

24. Mantovani A, Allavena P, Sica A. Tumour-associated macrophages as a prototypic type II polarised phagocyte population: role in tumour progression. Eur J Cancer. 2004;40(11):1660-7.

25. Biswas SK, Gangi L, Paul S, Schioppa T, Saccani A, Sironi M, et al. A distinct and unique transcriptional program expressed by tumor-associated macrophages (defective NF-\{kappa\}B and enhanced IRF-3/STAT1 activation). Blood. 2006;107(5):2112-22.

26. Laoui D, Van Overmeire E, Movahedi K, Van den Bossche J, Schouppe E, Mommer $C$, et al. Mononuclear phagocyte heterogeneity in cancer: different subsets and activation states reaching out at the tumor site. Immunobiology. 2011;216(11):1192-202.

27. Zaman MH, Trapani LM, Sieminski AL, MacKellar D, Gong H, Kamm RD, et al. Migration of tumor cells in 3D matrices is governed by matrix stiffness along with cell-matrix adhesion and proteolysis. Proc Natl Acad Sci U S A. 2006;103(29):10889-94.

28. Albini A, D'Agostini F, Giunciuglio D, Paglieri I, Balansky R, De Flora S. Inhibition of invasion, gelatinase activity, tumor take and metastasis of malignant cells by N-acetylcysteine. Int J Cancer. 1995;61(1):121-9.

29. Beyer M, Mallmann MR, Xue J, Staratschek-Jox A, Vorholt D, Krebs W, et al. High-resolution transcriptome of human macrophages. PLOS ONE. 2012;7(9), e45466.

30. Mantovani A. Macrophage diversity and polarization: in vivo veritas. Blood. 2006;108(2):408-9.

31. Porcheray F, Viaud S, Rimaniol A, Léone C, Samah B, Dereuddre-Bosquet N, et al. Macrophage activation switching: an asset for the resolution of inflammation. Clin Exp Immunol. 2005;142(3):481-9.

32. Chen $\mathrm{P}$, Bonaldo $\mathrm{P}$. Chapter one - role of macrophage polarization in tumor angiogenesis and vessel normalization: implications for new anticancer therapies. In: Kwang WJ, editor. International review of cell and molecular biology, vol. 301: Waltham, Massachusetts: Academic Press; 2013. p. 1-35.

33. Egeblad M, Werb Z. New functions for the matrix metalloproteinases in cancer progression. Nat Rev Cancer. 2002;2(3):161-74.

34. Bauvois B. New facets of matrix metalloproteinases MMP-2 and MMP-9 as cell surface transducers: outside-in signaling and relationship to tumor progression. Biochim Biophys Acta Rev Cancer. 2012;1825(1):29-36.

35. Morini M, Mottolese M, Ferrari N, Ghiorzo F, Buglioni S, Mortarini R, et al. The alpha 3 beta 1 integrin is associated with mammary carcinoma cell metastasis, invasion, and gelatinase B (MMP-9) activity. Int J Cancer. 2000;87(3):336-42.

36. Chang C, Werb Z. The many faces of metalloproteases: cell growth, invasion, angiogenesis and metastasis. Trends Cell Biol. 2001;11(11):S37-43.

37. Hagemann T. Enhanced invasiveness of breast cancer cell lines upon co-cultivation with macrophages is due to TNF-a dependent up-regulation of matrix metalloproteases. Carcinogenesis. 2004;25(8):1543-9.

38. Albini A, Melchiori A, Santi L, Liotta LA, Brown PD, Stetler-Stevenson WG. Tumor cell invasion inhibited by TIMP-2. J Natl Cancer Inst. 1991;83(11):775-9.

39. Foda HD, Zucker S. Matrix metalloproteinases in cancer invasion, metastasis and angiogenesis. Drug Discov Today. 2001;6(9):478-82
40. Mason SD, Joyce JA. Proteolytic networks in cancer. Trends Cell Biol. 2011;21(4):228-37.

41. Carmeliet P. Angiogenesis in life, disease and medicine. Nature. 2005:438(7070):932-6.

42. Knowles $H$, Leek $R$, Harris A. Macrophage infiltration and angiogenesis in human malignancy. Novartis Found Symp. 2004;256:189-200.

43. Coffelt SB, Hughes R, Lewis CE. Tumor-associated macrophages: effectors of angiogenesis and tumor progression. Biochim Biophys Acta Rev Cancer. 2009;1796(1):8-11.

44. Lin EY. Vascular endothelial growth factor restores delayed tumor progression in tumors depleted of macrophages. Mol Oncol. 2007;1:288-302.

45. Lin EY, Li J-F, Gnatovskiy L, Deng Y, Zhu L, Grzesik DA, et al. Macrophages regulate the angiogenic switch in a mouse model of breast cancer. Cancer Res. 2006;66(23):11238-46

46. Kodelja V, Muller C, Tenorio S, Schebesch C, Orfanos CE, Goerdt S Differences in angiogenic potential of classically vs alternatively activated macrophages. Immunobiology. 1997;197(5):478-93.

47. Jetten N, Verbruggen S, Gijbels M, Post M, Winther MJ, Donners MPC. Anti-inflammatory M2, but not pro-inflammatory M1 macrophages promote angiogenesis in vivo. Angiogenesis. 2013;17(1):109-18.

48. Rundhaug JE. Matrix metalloproteinases and angiogenesis. J Cell Mol Med. 2005;9(2):267-85.

\section{Submit your next manuscript to BioMed Central and take full advantage of:}

- Convenient online submission

- Thorough peer review

- No space constraints or color figure charges

- Immediate publication on acceptance

- Inclusion in PubMed, CAS, Scopus and Google Scholar

- Research which is freely available for redistribution 